\title{
Validation of the Publication of New Names and New Combinations Previously Effectively Published Outside the IJSB
}

\author{
List No. $62 \dagger$
}

The purpose of this announcement is to effect the valid publication of the following new names and new combinations under the procedure described previously [Int. J. Syst. Bacteriol. 27(3):iv, 1977]. Authors and other individuals wishing to have new names and/or combinations included in future lists should send the pertinent reprint or a photocopy thereof to the IJSB (c/o ASM) for confirmation that all of the other requirements for valid publication have been met. It should be noted that the date of valid publication of these new names and combinations is the date of publication of this list, not the date of the original publication of the names and combinations. The authors of the new names and combinations are as given below, and these authors' names will be included in the author index of the present issue and in the volume author index in this issue of the IJSB.

Inclusion of a name on these lists validates the name and thereby makes it available in bacteriological nomenclature. The inclusion of a name on this list is not to be construed as taxonomic acceptance of the taxon to which the name is applied. Indeed, some of these names may, in time, be shown to be synonyms, or the organisms may be transferred to another genus, thus necessitating the creation of a new combination.

\begin{tabular}{|c|c|c|c|c|}
\hline Name & Proposed as: & Author(s) (reference) & Priority ${ }^{a}$ & Nomenclatural type $^{b}$ \\
\hline Actinomadura latina & New species & Trujillo and Goodfellow (11) & 3 & Strain A10 (= DSM 43382) \\
\hline $\begin{array}{l}\text { Amycolatopsis japonica corrig. }{ }^{c} \\
\text { (Amycolatopsis japonicum [sic]) }\end{array}$ & New species & Goodfellow et al. (3) & 2 & Strain MG417-CF17 (= DSM 44213) \\
\hline $\begin{array}{l}\text { Azospirillum largomobile (basonym: } \\
\text { Conglomeromonas largomobilis subsp. } \\
\text { largomobilis) }\end{array}$ & New combination & Ben Dekhil et al. (1) & 1 & UQM 2041 (= ACM 2041) \\
\hline Bifidobacterium lactis & New species & Meile et al. (8) & 2 & Strain UR1 (= DSM 10140) \\
\hline Chromatium glycolicum & New species & Caumette et al. (2) & 5 & Strain SL $3201(=$ ATCC $700202=$ DSM 11080) \\
\hline Clostridium vincentii & New species & Mountfort et al. (9) & 7 & Strain lac-1 (= DSM 10228) \\
\hline Desulfotomaculum aeronauticum & New species & Hagenauer et al. (4) & 2 & Strain 9 (= DSM 10349) \\
\hline Ectothiorhodospira haloalkaliphila & New species & Imhoff and Süling (6) & 6 & Strain BN 9903 (= ATCC 51935) \\
\hline Ectothiorhodospira marina & New species & Imhoff and Süling (6) & 6 & Strain BA $1010(=241)$ \\
\hline Enterobacter kobei & New species & Kosako et al. (7) & 4 & Strain NIH 1485-79 (= JCM 8580) \\
\hline Halorhodospira & New genus & Imhoff and Süling (6) & 6 & Halorhodospira halophila \\
\hline $\begin{array}{l}\text { Halorhodospira abdelmalekii (basonym: } \\
\text { Ectothiorhodospira abdelmalekii) }\end{array}$ & New combination & Imhoff and Süling (6) & 6 & Strain BN 9840 (= DSM 2110) \\
\hline $\begin{array}{l}\text { Halorhodospira halochloris (basonym: } \\
\text { Ectothiorhodospira halochloris) }\end{array}$ & New combination & Imhoff and Süling (6) & 6 & DSM 1059 \\
\hline $\begin{array}{l}\text { Halorhodospira halophila (basonym: } \\
\text { Ectothiorhodospira halophila) }\end{array}$ & New combination & Imhoff and Süling (6) & 6 & DSM 244 \\
\hline Neisseria dentiae & New species & Sneath and Barrett (10) & 8 & Strain V33 (= Dent SHI/3848 = ATCC 700276) \\
\hline Sulfophobococcus & New genus & Hensel et al. (5) & 2 & Sulfophobococcus zilligii \\
\hline Sulfophobococcus zilligii & New species & Hensel et al. (5) & 2 & Strain K1 (= DSM 11193) \\
\hline
\end{tabular}

$\dagger$ Lists 1 through 61 were published in the Int. J. Syst. Bacteriol. 27:306, 1977; 29:79, 436, 1979; 30:601, 676, 1980; 31:215, 382, 1981; 32:266, 384, 1982; 33:438, 672, 896,$1983 ; \mathbf{3 4}: 91,270,355,503,1984 ; \mathbf{3 5}: 223,375,535, \mathbf{1 9 8 5} ; \mathbf{3 6}: 354,489,573,1986 ; \mathbf{3 7}: 179,1987 ; \mathbf{3 8}: 136,220,328,449,1988 ; \mathbf{3 9}: 93,205,371,495,1989 ; \mathbf{4 0}: 105,212$, 320, 470, 1990; 41:178, 331, 456, 580, 1991; 42:191, 327, 511, 656, 1992; 43:188, 398, 624, 864, 1993; 44:182, 370, 595, 852, 1994; 45:197, 418, 619, 879, 1995; 46:362, 625, 836, 1189, 1996; and 47:242, 601, 1997.

a Priority number assigned according to the date the documentation and request for validation are received.

${ }^{b}$ Abbreviations: ACM, Australian Collection of Microorganisms, Department of Microbiology, University of Queensland, Nathan, Brisbane, Australia; ATCC American Type Culture Collection, Rockville, Md.; DSM, DSMZ-Deutsche Sammlung von Mikroorganismen und Zellkulturen GmbH, Braunschweig, Germany; JCM, Japan Collection of Microorganisms, Riken, Saitama, Japan.

Name has been corrected in accordance with Rule 61 of the Bacteriological Code on the occasion of validation.

\section{REFERENCES}

1. Ben Dekhil, S, M. Cahill, E. Stackebrandt, and L. I Sly. 1997. Transfer of Conglomeromonas largomobilis subsp. largomobilis to the genus Azospirillum as Azospirillum largomobile comb. nov., and elevation of Conglomeromonas largomobilis subsp. parooensis to the new type species of Conglomeromonas, Conglomeromonas parooensis sp. nov. Syst. Appl. Microbiol. 20:72-77.

2. Caumette, P., J. F. Imhoff, J. Süling, and R. Matheron. 1997. Chromatium glycolicum sp. nov., a moderately halophilic purple sulfur bacterium that uses glycolate as substrate. Arch. Microbiol. 167:11-18.

3. Goodfellow, M., A. B. Brown, J. Cai, J. Chun, and M. D. Collins. 1997. Amycolatopsis japonicum sp. nov., an actinomycete producing $(\mathrm{S}, \mathrm{S})-\mathrm{N}, \mathrm{N}^{\prime}$. ethylenediaminedisuccinic acid. Syst. Appl. Microbiol. 20:78-84.
4. Hagenauer, A., H. Hippe, and F. A. Rainey. 1997. Desulfotomaculum aeronauticum sp. nov., a sporeforming, thiosulfate-reducing bacterium from corroded aluminium alloy in an aircraft. Syst. Appl. Microbiol, 20:65-71.

5. Hensel, R., K. Matussek, K. Michalke, L. Tacke, B. J. Tindall, M. Kohlhoff, B. Siebers, and J. Dielenschneider. 1997. Sulfophobococcus zilligii gen. nov., spec. nov. a novel hyperthermophilic archaeum isolated from hot alkaline springs of Iceland. Syst. Appl. Microbiol, 20:102-110.

6. Imhoff, J. F., and J. Süling. 1996. The phylogenetic relationship among Ectothiorhodospiraceae: a reevaluation of their taxonomy on the basis of 16S rDNA analyses. Arch. Microbiol. 165:106-113.

7. Kosako, Y., K. Tamura, R. Sakazaki, and K. Miki. 1996. Enterobacter kobei sp. nov., a new species of the family Enterobacteriaceae resembling Enter- 
obacter cloacae. Curr. Microbiol. 33:261-265.

8. Meile, L., W. Ludwig, U. Rueger, C. Gut, P. Kaufmann, G. Dasen, S. Wenger, and M. Teuber. 1997. Bifidobacterium lactis sp. nov., a moderately oxygen tolerant species isolated from fermented milk. Syst. Appl. Microbiol. 20:57-64.

9. Mountfort, D. O., F. A. Rainey, J. Burghardt, H. F. Kaspar, and E. Stackebrandt. 1997. Clostridium vincentii sp. nov., a new obligately anaerobic, saccharolytic, psychrophilic bacterium isolated from low-salinity pond sedi- ment of the McMurdo Ice Shelf, Antarctica. Arch. Microbiol. 167:54-60.

10. Sneath, P. H. A., and S. J. Barrett. 1996. A new species of Neisseria from the dental plaque of the domestic cow, Neisseria dentiae sp. nov. Lett. Appl. Microbiol. 23:355-358.

11. Trujillo, M. E., and M. Goodfellow. 1997. Polyphasic taxonomic study of clinically significant actinomadurae including the description of Actinomadura latina sp. nov. Zentralbl. Bakteriol. 285:212-233. 